\title{
Article Withdrawn
}

\section{Article withdrawn 'Biochemical and phytochemical study on Nannochloropsis oculata extract against alterations in spleen of streptozotocin induced diabetic rats'.}

Article titled 'Biochemical and phytochemical study on Nannochloropsis oculata extract against alterations in spleen of streptozotocin induced diabetic rats' which was published earlier as 'online first' has been withdrawn from the journal in compliance with the request received from authors of the manuscript. 